\section{One size does not fit all: trans-diagnostic immune signatures for personalized treatment of psychoses}

Braz J Psychiatry. 2022 Mar-Apr;44(2):218-219 doi:10.1590/1516-4446-2021-2302

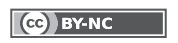

Immune dysregulation has been widely implicated in the pathophysiology of schizophrenia and related psychoses, as well as in depression and other psychiatric disorders. ${ }^{1-3}$ Meta-analyses consistently show elevated levels of pro-inflammatory cytokines in the blood and cerebrospinal fluid of patients during a first episode and before drug treatment. Longitudinal studies suggest that inflammation in childhood/adolescence precedes the onset of psychoses and depression in adulthood. Large Mendelian randomization studies, which use genetic variants that regulate the levels/activity of a biomarker, have provided compelling evidence that inflammation, in particular interleukin-6, is unlikely a consequence of residual confounding or reverse causation and may be causally related to both psychoses and depression. The reasons for the apparent trans-diagnostic effect of inflammation are still obscure, but it is possible that inflammation is a biological mechanism for symptoms commonly shared across psychiatric disorders.

Approximately one-third of patients do not respond to conventional monoamine drug treatment, and a similar proportion has signs of inflammation in the blood that predict poor treatment response. Targeting the immune system may thus be promising for innovative treatment strategies. However, an important paradoxical finding is that large clinical trials have generally failed to show the benefit of adjunctive anti-inflammatory treatments. Notably, a recent study reported that minocycline, an antibiotic that inhibits the inflammation of microglia (resident immune cells in the brain), was as ineffective as adjunctive therapy for schizophrenia. ${ }^{4}$

A potential explanation for the null findings is that previous trials were focused on categorical diagnosis and the "one size fits all" approach to treating heterogeneous syndromes. Since inflammation is likely to occur only in a subset of patients, conventional frameworks are too reductionist to transcend traditional diagnostic boundaries and characterize heterogeneous and trans-diagnostic symptom profiles. Innovative approaches, such as the Research Domain Criteria and the Hierarchical Taxonomy of Psychopathology, propose a shift from single disease entities towards trans-diagnostic dimensions of symptoms and multifactorial constructs that map within and across many disorders. ${ }^{5}$ Recent research ${ }^{6}$ raises the possibility that features occurring in several psychiatric disorders share a common inflammatory substrate unrelated to diagnosis. Emerging investigation ${ }^{3}$ has begun to apply symptom-based approaches and Mendelian randomization to test the specificity and causality of associations, unveiling specific symptoms underpinned by trans-diagnostic inflammatory mechanisms, particularly anhedonia, somatic/neurovegetative symptoms (fatigue, appetite/ sleep disturbance), and cognitive impairment. Such advanced approaches may provide significant insight into immune pathogenesis and inform more homogeneous sample selection for future clinical trials.

Identifying biomarkers that transcend current diagnostic limits to translate the complex neurobiology of transdiagnostic symptoms/domains will be challenging but holds the potential to move psychiatry forward to successful personalized treatment. ${ }^{6}$ For example, low counts of blood $\mathrm{T}$ regulatory cells (a subpopulation of lymphocytes that maintain immune homeostasis) correlate with more negative and cognitive symptoms in some patients with psychoses $^{2}$. Future trials aiming to boost Tregs in blood should target patients with primary negative/cognitive symptoms and consider deep immuno-phenotyping of peripheral blood immune cells combined with conventional cytokine assays and multimodal neuroimaging integrated into a symptom-network. ${ }^{2,6}$ Biomarker-based machine learning is beginning to disentangle disease heterogeneity, identify comorbid trans-diagnostic symptoms, and reliably classify patients based on complex immunological features that could affect treatment decisions but may be hidden within conventional categorical diagnoses. $^{7}$ These will be key to identifying transdiagnostic immune signatures and patients with signs of immune dysregulation (who are more likely to benefit from adjunctive anti-inflammatory treatments) for inclusion in future personalized trials.

Fabiana Corsi-Zuelli iD

Departamento de Neurociências e Ciências do Comportamento, Departamento de Psiquiatria, Faculdade de Medicina de Ribeirão Preto, Universidade de São Paulo (FMRP/USP), Ribeirão Preto, São Paulo, Brazil.

Submitted Oct 13 2021, accepted Dec 31 2021, Epub Mar 072022.

\section{Acknowledgements}

FC-Z acknowledges national and international doctorate fellowships from Fundação de Amparo à Pesquisa do Estado de São Paulo (FAPESP \#2019/13229-2 and \#2021/07448-3). This letter was written as part of a student evaluation at the Faculdade de Medicina, Universidade de São Paulo (FMUSP) graduate course called "Transtornos mentais a partir de neurocircuitos: uma nova abordagem para a psiquiatria" (MPS5819-2/1), coordinated by Drs. Elizabeth Shepard, Euripedes Constantino Miguel Filho, Hermano Tavares, Marcelo Camargo Batistuzzo, and Marcelo Queiroz Hoexter. FC-Z acknowledges the course coordinators and the professors, researchers, and students who took part in this course for the fruitful discussion that stimulated this letter. FC-Z would also like to thank Prof. Bill Deakin (University of Manchester) and Prof. Rachel Upthegrove 
(University of Birmingham) for reading this letter and providing helpful feedback.

\section{Disclosure}

The author reports no conflicts of interest.

How to cite this article: Corsi-Zuelli F. One size does not fit all: trans-diagnostic immune signatures for personalized treatment of psychoses. Braz $\mathrm{J}$ Psychiatry. 2022;44:218-219. http://dx.doi.org/10.1590/1516-44462021-2302

\section{References}

1 Corsi-Zuelli F, Deakin B. Impaired regulatory T cell control of astroglial overdrive and microglial pruning in schizophrenia. Neurosci Biobehav Rev. 2021;125:637-53.
2 Corsi-Zuelli F, Deakin B, de Lima MH, Qureshi O, Barnes NM, Upthegrove $R$, et al. $T$ regulatory cells as a potential therapeutic target in psychosis? Current challenges and future perspectives. Brain Behav Immun Health. 2021;17:100330.

3 Milaneschi Y, Kappelmann N, Ye Z, Lamers F, Moser S, Jones PB, et al. Association of inflammation with depression and anxiety: evidence for symptom-specificity and potential causality from UK Biobank and NESDA cohorts. Mol Psychiatry. 2021 Jun 16. doi: 10.1038/ s41380-021-01188-w. Online ahead of print.

4 Deakin B, Suckling J, Barnes TR, Byrne K, Chaudhry IM, Dazzan P, et al. The benefit of minocycline on negative symptoms of schizophrenia in patients with recent-onset psychosis (BeneMin): a randomised, double-blind, placebo-controlled trial. Lancet Psychiatry. 2018;5:885-94.

5 Michelini G, Palumbo IM, DeYoung CG, Latzman RD, Kotov R. Linking RDoC and HiTOP: a new interface for advancing psychiatric nosology and neuroscience. Clin Psychol Rev. 2021;86:102025.

6 MacKenzie G, Subramaniam S, Caldwell LJ, Fitzgerald D, Harrison NA, Hong $S$, et al. Research priorities for neuroimmunology: identifying the key research questions to be addressed by 2030 . Wellcome Open Res. 2021;6:194. 